\title{
Current issues of personnel and laboratory practices in genetic testing
}

Hon Fong Louie Mark, Thaddeus Kelly, Michael S Watson, Gerald Hoeltge, Wayne A Miller, Laurent Beauregard

\begin{abstract}
As genetic testing is an area with implications extending far beyond that of the primary patient, it is appropriately an area that is under increased scrutiny. To ensure that high quality is maintained in the delivery of genetic services, several agencies have developed standards and guidelines. The present article summarises important recommendations made by the American College of Medical Genetics (ACMG), the College of American Pathologists (CAP), the US Health Care Financing Administration (HCFA), and the US Food and Drug Administration (FDA) as they relate to genetic testing. Some of the standards are based on voluntary compliance, whereas others have the force of regulation. They all address issues of personnel credentials, laboratory operations, and the most critical quality assurance and control measures for diagnostic laboratories from the perspective of various agencies. In most instances, the standards promulgated by these agencies are offered as minimum criteria. The exact impact of these regulations on the practice of medical genetics has yet to be established.
\end{abstract}

(f Med Genet 1995;32:780-786)

Genetic testing is undoubtedly a topic with many broad implications that affect the interests of the primary patient. Accordingly, all genetic testing laboratories are expected to operate under the guidance of standards that will ensure a high quality of service. Recently, several agencies and professional organisations have developed standards which are applicable to genetic services. These include the American College of Medical Genetics (ACMG), the College of American Pathologists (CAP), the US Health Care Financing Administration (HCFA), and the US Food and Drug Administration (FDA). Some of the standards are based on voluntary compliance, whereas others have the force of regulation. They all address issues of personnel certification and laboratory operations. In most instances, the promulgated standards are offered as minimum criteria covering various facets of genetic testing.

The responsibility for certifying qualified medical geneticists and for accrediting training programmes rests with the American Board of Medical Genetics (ABMG). The ABMG has recently been recognised as the 24th primary specialty board of the American Board of Med- ical Specialties (ABMS). As a result, the $A B M G$ is currently formulating policies for recertification of its diplomates as a means of indicating continuing proficiency in medical genetics.

The present article aims to provide an overview of the state of the standards and to define the issues of quality in the delivery of laboratory genetic services.

The discipline of medical genetics, genetic testing laboratories, and personnel standards for medical geneticists

Before 1960, the discipline of genetics did not exist in the United States as a recognised area of specialisation in the practice of medicine. Genetics was an academic, "ivory tower" topic far removed from inpatient wards and outpatient clinics. In the early 1960 s, several areas began to converge into the specialty of medical genetics. There were specific advances which made this process possible. The use of mitogens ${ }^{1}$ to effect the culture of peripheral blood lymphocytes led to the rapid development of cytogenetic laboratories at academic medical centres. The early clinical laboratory geneticists in the US were mainly MDs. The application of increasingly sophisticated biochemical analysis to inborn errors of metabolism began to suggest a clinically relevant means for management. The field of biochemical genetics owed its early development to Guthrie's development of a bacterial inhibition assay for newborn screening of PKU. ${ }^{2}$ The sensitivity and specificity of this test served as a basis for the screening programme which followed. Finally, with the development of polio vaccines, the change in emphasis on the part of the March of Dimes to "fight birth defects" opened opportunities for the study of birth defects and syndromes as advanced by David Smith. ${ }^{3}$ The term "funny looking kid (FLK)" was forever replaced by "dysmorphology".

It was in the early 1970 s that medical genetics began to emerge as a medical discipline as a result of the proliferation of fellowship training programmes, notably that of $\mathrm{Dr}$ Victor $\mathrm{McKu}-$ sick at Johns Hopkins. While there were many developments in the $1980 \mathrm{~s}$, three were most notable as they related to the development of the specialty of medical genetics. The ABMG was formed in 1980 under the sponsorship of the American Society of Human Genetics (ASHG). The first certifying examination was given in 1981, and commercial laboratories, especially those in cytogenetics, began to ap- 
ABMG certificates by examination: five examination cycles are shown

\begin{tabular}{|c|c|c|c|c|c|c|}
\hline Specialty & 1982 & 1984 & 1987 & 1990 & 1993 & Totals \\
\hline $\begin{array}{l}\text { Biochemical } \\
\text { Cytogenetics } \\
\text { Counselling } \\
\text { PhD MG } \\
\text { Molecular } \\
\text { Clinical } \\
\text { Biochem/Mol }\end{array}$ & $\begin{array}{r}57 \\
123 \\
167 \\
56\end{array}$ & $\begin{array}{r}26 \\
79 \\
144 \\
30\end{array}$ & $\begin{array}{r}25 \\
103 \\
179 \\
27\end{array}$ & $\begin{array}{r}134 \\
47 \\
398\end{array}$ & $\begin{array}{r}29 \\
61 \\
182 \\
12 \\
144 \\
136 \\
2 \\
563\end{array}$ & $\begin{array}{r}137 \\
427 \\
814 \\
137 \\
144 \\
794 \\
49 \\
2501\end{array}$ \\
\hline
\end{tabular}

pear and flourish. It was also during the 1980s that the polymerase chain reaction was born in the redwood forests of California.

With the arrival of the 1990 s came the recognition of the ABMG as the 24th primary specialty board of the ABMS. Professional organisations representing medical geneticists have been restructured into a tripartite structure characteristic of most medical specialties. The American Board of Medical Genetics (ABMG) is the accreditation body for training programmes and the certifying board for medical geneticists. The American College of Medical Genetics (ACMG), founded in 1991, is the standards and practice arm of the medical genetics community while the parent organisation, the American Society of Human Genetics (ASHG), is now the scientific research arm of the field.

The certificates issued each year by the ABMG by specialty are listed in the table. As can be seen from this, there was a large reservoir of practising geneticists when the first round of certificates was issued in 1982. Since then, one can see that there has been a plateau or a slight decrease in each specialty. This can also be depicted by the pie chart shown in the figure. About one-third of certified people are counsellors, one-third clinical geneticists, and the other specialties account for the final onethird. A list of ABMG certified people by specialty and by state may be obtained by consulting the Official ABMS Directory of Board Certified Medical Specialists (the 27th edition has been released in 1995).

Interesting observations can be drawn from the 1993 certifying examinations. Of 581 ex-

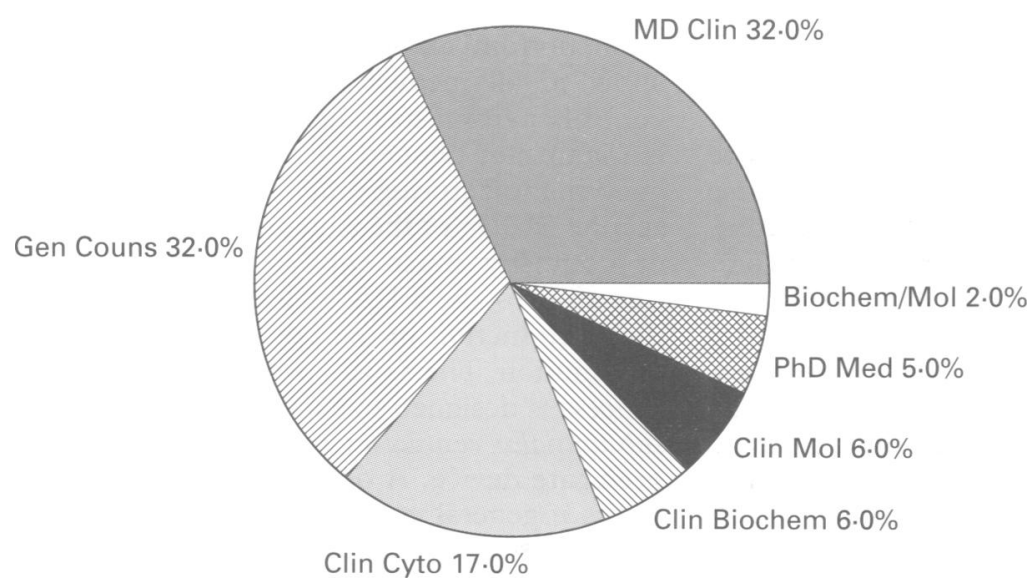

Distribution of certified medical genetics personnel. MD Clin are MD clinical geneticists. Biochem/Mol are biochemical and molecular geneticists. PhD Med are PhD medical geneticists. Clin Mol are clinical molecular geneticists. Clin Biochem are clinical biochemical geneticists. Clin Cyto are clinical cytogeneticists. Gen Couns are genetic counsellors. aminations administered, there were 385 (66\%) passing grades. This varied from a low of $51 \%$ in Clinical Cytogenetics to a high of $73 \%$ in Clinical Genetics. Pass/fail grades were not determined by a predefined percentage score. Rather, every effort was made to establish a minimum score that corresponded to an acceptable level of competence in the field. One major reason for the high failure rate in Clinical Cytogenetics was the poor performance of the group on the General Examination. It was noted that genetic counsellors outscored cytogeneticists on the cytogenetics portion of the General Examination! Overall, the examinations showed that people who had inadequate training in formal genetics had great difficulty in passing the examinations. This naturally leads one to question whether medical genetics represents an assortment of technical skills or whether it encompasses a body of knowledge applicable to medical practice. The ABMG clearly thinks that the latter is the case and therefore will continue to stress formal genetics in its examinations. In the immediate future, several changes will be inaugurated by the ABMG. First, clinical genetic training programmes will be accredited by the American Council for Graduate Medical Education (ACGME). Second, for a centre to offer training in any other ABMG specialty, the centre must first be accredited by the ACGME. Third, $\log$ books for all specialties will require 150 cases. Fourth, a third year of training will be required of people seeking certification in a second specialty. This is strongly encouraged for 1996 and will be mandatory for 1999 .

The current distribution of certified medical genetics personnel is shown in the figure. Many of these people currently serve as directors in genetic testing laboratories.

\section{Standards of practice for genetic testing laboratories}

In recognition of the broad implications of genetic testing and the critical need for high standards and self-regulation, the American College of Medical Genetics (ACMG) finalised and endorsed, on 21 March 1994, the document entitled Standards and Guidelines: Clinical Genetics Laboratories. ${ }^{4}$ This document reflects the consensus of the medical genetics community on standards of genetics laboratory practices. It was recognised that a fundamental body of knowledge of human genetics was the unifying factor among medical geneticists and that this knowledge was critical to the performance of genetic testing and the subsequent interpretation of test results. Hence, the standards reflect issues common to all genetics laboratories as well as those that are specific to each of the laboratory specialties. This publication, also discussed in Mark and Watson, ${ }^{5}$ consists of the following major subsections: overview, personnel policies, general policies, shared methodologies, clinical cytogenetics, clinical biochemical genetics, and clinical molecular genetics. By mid 1995 the standards will be expanded to encompass a major section on prenatal biochemical genetic 
screening and several subsections reflecting new areas of testing within each specialty.

Despite the efforts of the professional community to develop such standards of practice, it has become increasingly difficult to balance the slowly evolving regulatory constraints in this area with the rapid advances in genetic testing. To a large degree, this reflects the relatively high technology used in this field, the esoteric nature of the testing, and the lack of people appropriately trained to oversee testing independently.

To facilitate the transition of new tests and technologies into service, the ACMG Standards and Guidelines will begin to include the laboratory test validation component of test development and provision. Similarly, the College will solicit from its membership specific data on the controls used for validation with the ultimate goal being to collect population based data on the use of particular DNA probes in normal people. Similarly, to provide a method for verifying that the probes received are the probes which were ordered, a cell line resource for the internal validation of new tests is being developed for all laboratories. This will be applicable to both manufactured probes and "home brew" probes. Hence, even in the absence of FDA approval for in vitro diagnostic devices, a laboratory can internally confirm both the expected localisation of the probe and the expected sensitivity of the assay.

In contrast to the regulations of the products used in genetic testing, which are manufactured and sold over state lines and, hence, under the jurisdiction of the FDA, there are also the regulations which govern laboratory practices. These practices are regulated through CLIA ' 88 which is a function of HCFA. Again, the problem arises from having high complexity tesing practices that are monitored through a system that is ill equipped to evaluate the competence of individual laboratories. To address this problem, the ACMG and the CAP (an agency with deemed status) have identified a significant number of appropriately trained and experienced people across the US to perform laboratory inspections. A list of these people can be obtained from the ACMG.

While activities that apply to genetic testing services are being developed at the federal level, the states have continued to fill the perceived void. At least nine states have now developed regulations which are either equally or more stringent than those at the federal level. These states include California, Oregon, Washington, Idaho, Florida, Ohio, New Jersey, New York, and Maryland. Other states, such as Rhode Island, have formally endorsed the ACMG Standards and Guidelines. In the current environment of shifting responsibilities from the federal to the state level, this trend is likely to continue.

While the above comments have focused on the laboratory component of testing, it is equally important to consider the programmatic nature of genetic testing. It is recognised that the majority of errors related to laboratory testing occurs in the preanalytic and postanalytic phases of testing. Hence, errors tend to involve inappropriate, incorrect, or totally absent test requests in the preanalytic phase and poor communication of test results to the physician and to the patient in the postanalytic phase. Much of this reflects the lack of previous exposure to human genetics and medical genetics offered to medical students and clinicians in the past and is a problem not easily resolved. Clearly, extensive efforts towards continuing medical education, such as the programmes sponsored annually by the ACMG jointly with the March of Dimes, will be required.

\section{Federal regulations for diagnostic genetic testing: the FDA perspective} Any type of medical device destined for interstate commerce is regulated by the US Food and Drug Administration (FDA). Oversight of this regulation process falls primarily with the FDA's Center for Devices and Radiological Health (CDRH). Any in vitro diagnostic devices, such as those used to test for human genetic disorders, are reviewed with the Center's Division of Clinical Laboratory Devices in the Office of Device Evaluation. The FDA's authority to regulate medical devices is based on several legislative acts and amendments, namely the Federal Food, Drug and Cosmetic Act of 1938, the Medical Device Amendments of 1976, and the Safe Medical Devices Act (SMDA) of 1990.

The FDA defines a medical device as “. . . any instrument, apparatus, machine, implant, in vitro reagent, or other similar article . . . that is intended for use in the diagnosis of disease or other conditions (such as pregnancy), or in the cure, mitigation, treatment or prevention of disease in man or other animals ..." (21 CFR 201 (h)). With the implementation of the Medical Device Amendment of 1976, the FDA is directed to classify all medical devices that are in commercial distribution. These devices are classified into one of three regulatory categories: class I, II, or III. The intent of this classification system is to provide appropriate levels of regulation with regard to safety and effectiveness of these devices.

Class I devices require the least amount of regulation and fall under general controls which apply to all medical devices. These general controls pertain to issues of misbranding, the marketing of banned devices, and the use of good manufacturing practices (GMPs). Under section $510(\mathrm{k})$ of the Act, an individual person or company must submit a premarket notification to the FDA at least 90 days before the introduction of the device for commercial distribution. The $510(\mathrm{k})$ premarket notification process is designed to establish that a device is substantially equivalent to a legally marketed, predicate device. A device is granted a class I status if general controls are sufficient to provide reasonable assurance of safety and effectiveness.

To provide reasonable assurance of safety and effectiveness, class II medical devices are also evaluated under Section $510(\mathrm{k})$ and include those devices for which general controls 
are insufficient. Additional controls needed for class II devices may include performance standards, postmarket surveillance, and guidance for submission of clinical data.

Class III devices are those in which insufficient information exists to assure that the aforementioned controls will provide reasonable safety and effectiveness. In addition to class I and II controls, class III devices require premarket approval (PMA), a lengthy and stringent process designed to establish intrinsic safety and effectiveness of the device, rather than comparable safety and effectiveness as with a class I or II device.

Most medical devices intended for in vitro diagnostic (IVD) use, such as DNA probes used for fluorescent in situ hybridisation, require PMA approval or $510(\mathrm{k})$ clearance by the FDA before the device can be legally marketed. The purpose of a $510(\mathrm{k})$ clearance is to establish that a device is substantially equivalent to a legally marketed existing device. To do this, the manufacturer must provide evidence that the new device has the same intended use and shares the same technological characteristics and performance characteristics provided that the new device can be shown to be as safe and effective as the predicate device. The $510(\mathrm{k})$ process is required for most class I, all class II, and a few preamendment class III devices.

The second major pathway for introducing a device into the marketplace is through the premarket approval (PMA) process. As noted, this process is intended to establish the intrinsic safety and effectiveness of a medical device, rather than comparable characteristics. The PMA is the most stringent of the CDRH's premarket evaluation process and requires a longer review time (180 days versus 90 days for $510(\mathrm{k})$ clearance), proof of good manufacturing practices via on site inspections, and a review by an expert advisory panel. The use of such an advisory panel gives the FDA the ability to ask appropriate questions as well as identify critical performance parameters necessary for a given device. Since the PMA process is more involved than the $510(\mathrm{k})$ process, it becomes more costly to the manufacturer, the FDA, and ultimately the consumer.

A medical device's regulatory status is indicated by the product's labelling. If a product is labelled "For in vitro diagnostic use", the device has either been cleared by the FDA through the $510(\mathrm{k})$ process or approved by the FDA through the PMA process. Any patient test result obtained from using such a device may be reported as reflective of that device's intended use. Medical devices that are not FDA cleared or approved are labelled in one of the following ways: (1) "For research use only. Not for use in diagnostic procedures." This indicates that the product does not have a special clinical or diagnostic claim. Consequently, any test result obtained from such devices should not be reported or used for establishing test performance characteristics; (2) "For investigational use only. The performance characteristics of this product have not been established." Investigational devices are considered to be in a presubmission status.
All investigational use is presumed to be for the collection of data to support a clinical utility claim. In many instances, the use of such a product may require an Institutional Review Board (IRB) approval which usually requires informed patient consent.

Most in vitro devices currently used in genetic testing are labelled for "research" or "investigational" use; few have been FDA cleared or approved for "diagnostic" use. The FDA is aware that many so called "research" devices have been in extensive clinical use for a significant period of time. In order to address the concerns of inappropriate commercialisation of in vitro devices, the FDA is developing a Compliance Policy Guide that will establish guidance to enable manufacturers of devices that are currently being used or commercialised for diagnostic purposes or procedures to achieve compliance with the regulations that apply to in vitro diagnostic devices. The FDA recognises the potential detrimental effect on the standards of clinical practice if immediate regulatory actions are taken against certain unapproved/uncleared devices that have been commercially marketed. In order to implement compliance with the regulations without causing undue disruption to the possible beneficial use, the FDA, based on its risk assessment of potential harm and benefit to the public health, ${ }^{6}$ may under certain conditions permit manufacturers a period of grace to submit documents seeking FDA clearance or approval. Full details of the document are expected to be released within the next three to six months. ${ }^{7}$

Most genetic devices are new (postamendment) device types and are by default class III requiring a PMA review before marketing. In reality, this level of review (PMA) may not be necessary to assure the safe and effective use of all categories of new products. For some products, general controls or special controls and a premarket evaluation under $510(\mathrm{k})$ may be appropriate. However, such an approach may require classification or reclassification of certain device types or implementation of new regulations, all of which are lengthy and cumbersome processes. In order to facilitate appropriate commercialisation of diagnostic devices, the FDA is exploring potential applications of existing statutes and regulations as well as evaluating the need for new regulations.

\section{Quality control/quality assurance issues for genetic testing laboratories: the CAP and HCFA perspectives}

The CAP offer several programmes to assist laboratories in achieving their quality control and improvement goals. Chief among these are the Laboratory Accreditation Program (LAP) and the Interlaboratory Comparison (Surveys) Program.

The LAP is the largest voluntary peer review programme in the world. Consisting of combined on site inspections and proficiency testing, it currently accredits over 4800 laboratories. The LAP, which has been deemed by the HCFA as a certifying agency under the Clinical Laboratory Improvement Amend- 
ments of 1988 (CLIA '88), is also recognised by the Joint Commission on Accreditation of Healthcare Organizations and many state agencies. The CAP's Standards for Laboratory Accreditation define the principles of the programme. More than 3500 checklist questions are used for field evaluation.

Participation in an appropriate proficiency testing (PT) programme, while insufficient by itself for certifying laboratory quality, is required for accreditation by the College whenever an appropriate survey is available. Proficiency testing provides an external benchmark indicator that can be used to monitor the performance of a laboratory between on site inspections. CAP surveys are designed to be educational instruments for laboratory improvement, and share five characteristics. First, they have been authored by respected experts in the field. Second, all participants receive the same survey material. Third, multiple challenges are offered, usually three times a year. Fourth, objective responses are required. Fifth, specimens that are issued for evaluation simulate real clinical situations.

The first PT (Interlaboratory Comparison Program) challenge offered by the CAP in genetics was Survey CY (Cytogenetics) in $1986 .{ }^{8}$ The American Society of Human Genetics joined as a cosponsor in 1991, and cosponsorship transferred to the American College of Medical Genetics in 1994. Survey CY has a subscription level of over 330 laboratories among 14 countries. About 270 are regular participants. Survey CY consists of three mailings per year. Each mailing contains four photographic challenges provided as offset prints (graded), either a peripheral blood sample or an incorrectly arranged karyotype (graded), interpretive questions (ungraded), and supplementary questions that seek to define a participant's needs and the state of the art (optional). Over half of the challenges are from peripheral blood lymphocytes, about $20 \%$ are taken from cultures of amniotic fluid cells or conceptional tissue, and the remainder are bone marrow and other neoplastic challenges. ${ }^{9}$

Grading is by consensus. At least $80 \%$ of the participants or referee laboratories must agree for a challenge to be evaluable. Decisions regarding acceptable level of precision for a response and synonymy are made by the authoring resource committee. Referee laboratories are chosen from among the participants on the basis of past superior performance.

In the past, four attributes of each case were scored independently: recognition of chromosomal abnormalities; sex chromosome designation; modal chromosome number; and proper use of An International System for Cytogenetic Nomenclature (ISCN, 1985, 1991)..$^{11}$ This required participants to use cumbersome and artificial scoring lists unlike a real clinical situation. Beginning in 1995, participants will only need to submit a karyotypic designation and the four attributes will be inferred from the text.

Survey CY, like all CAP surveys, is structured according to the needs of its participants. In the past, subscribers have reported problems with the quality of the photographic materials, poorly designed questionnaires, delayed shipments to the laboratory, and leaking vials. Each of these problems has been addressed directly. Late returns of questionnaires from the field and illegibility of written responses remain occasional problems identified by the CAP staff.

Two additional genetics surveys are offered. Each is cosponsored by the American College of Medical Genetics. Survey BGL (Biochemical Genetics), which began in 1993, currently has over 50 participants. BGL offers challenges in amino acid analysis, organic acid analysis, and mucopolysaccharide analysis. Next year Survey MGL will be inaugurated. MGL will include dystrophin gene analysis, mutation analysis (cystic fibrosis and haemoglobinopathy), fragile $\mathrm{X}$ analysis, and linkage analysis by Southern blotting. Both BGL and MGL stress interpretative skills.

All proficiency testing (Interlaboratory Comparison Program) samples should be handled as though they were real clinical material. Analysts need not be blinded, but they must be cautioned to follow the routine procedure for testing. CAP survey challenges may not be discussed with other laboratories nor referred to another laboratory for testing. These practices are mandated under CLIA ' 88 for those analytes regulated under the stature. Currently, none of the analytic challenges in CY, BGL, or MGL is regulated by HCFA, but cytogenetic and biochemical genetic laboratories seeking accreditation by the CAP must participate in the relevant surveys. "Participation" means that the laboratory reports results to the Surveys Program regularly for evaluation. Because these particular analytes are unregulated by HCFA, no minimum score is required. Accredited laboratories, however, must document internal review of every response graded as unacceptable along with their corrective actions.

\section{Concluding comments}

The rapid pace of new developments with potential application in the field of medical genetics is placing great pressures on clinical laboratories to implement new procedures quickly and to adopt new test systems for diagnostic purposes. How laboratories will respond to these pressures is of growing concern to medical geneticists who provide the services and to the regulators who have a responsibility to protect the public interest. The goal of both constituencies is to provide accurate and timely laboratory services to patients and their families. The purpose of this discussion is to review the new developments from the perspective of each constituency and to identify a common ground which is likely to lead to the attainment of the mutually held goal.

The discussion focused on three principal topics. They include (1) training and certification of personnel, (2) evaluation of the performance of the laboratory and its staff, and (3) validation of test systems.

A review of the 1994-1995 Guide to North American Graduate and Postgraduate Training Programs in Human Genetics ${ }^{12}$ indicates that 
there are 114 institutions which describe themselves as providing some aspect of training in human genetics. One hundred institutions are in the United States and 72 of these are accredited or have pending accreditation as training sites granted by the American Board of Medical Genetics. With the recognition of the ABMG as a primary specialty board of the ABMS, several changes in the accreditation process are being implemented. In the future, clinical genetic training programmes will be accredited by the American Council for Graduate Medical Education (ACGME). The ABMG will continue to provide accreditation for training programmes in the other medical genetic subspecialties but centres offering training in these other specialties must first be accredited by the ACGME. Because of these new requirements, it is likely that the number of accredited training sites in this country will decrease in the future.

The results of the 1993 ABMG certifying examinations points out a potential area of concern for the training programmes. The relatively poor performance on the General Examination among some of the subspecialty applicants supports the need for improved training in formal genetics. It is expected that the ABMG will continue to require a broad based knowledge of genetics and that the training concerns will be addressed in the accreditation process. In addition, a plan for recertification is currently being developed by the Recertification Committee of the ABMG and should be implemented by the time of the next examination cycle.

Based on current experience in fellowship programmes and the results of ABMG certifying examinations, it is possible to make some predictions about future needs for physician clinical geneticists. The predictions are based on several assumptions. At least one clinical genetics unit, staffed by three clinical geneticists, is needed per million population. The US population should be served by 1000 geneticists of whom there are currently more than 700 (table). Training programmes graduate approximately 43 clinicians per year and each year 23 are expected to retire. At that rate, personnel needs will not be reached until approximately the year 2010 . The potential limitation of this prediction is that major changes in the delivery of genetic services are likely. The changes will include an increased emphasis on managed care delivery systems and recognition of a genetic basis for many common diseases. The influence of these two factors on clinical genetics remains to be seen.

In the United States, genetic testing is offered in several venues. The primary sites include hospital based laboratories, research laboratories which are often in medical school settings, and commercial laboratories some of which limit their services to genetic testing. There are no reliable data which describe the full range of operating laboratories. The 1994 International Cytogenetic Laboratory Directory ${ }^{13}$ lists 320 laboratories in the US which describe themselves as providing genetic laboratory services. In response to a 1993 sur- vey, 274 of these laboratories indicated that they had completed 595632 tests, most of which were chromosome analyses. It is not yet possible to estimate what proportion of the total US genetic test volume is represented by this number.

The ACMG, founded in 1991, has assumed responsibility for developing standards of practice for the medical genetics community. The recently published Standards and Guidelines: Clinical Genetics Laboratories ${ }^{4}$ is expected to be an evolving document which reflects the consensus of participating ACMG members. Adherence to the listed standards will provide laboratory directors with the opportunity to offer a satisfactory level of genetic testing and will provide laboratory clients with some assurance that ordered tests are consistent with current standards of practice. It should be emphasised, however, that the guidelines present a minimal acceptable standard. Individual laboratory directors may establish more rigorous standards within their own programmes.

Adherence to at least minimal standards of laboratory practice is assured by external review. Laboratory certification by HCFA under authority of CLIA '88 includes regulations which are specific to genetic testing. The CAP provides a voluntary approach to fulfilling HCFA requirements. The CAP in collaboration with ACMG is working to improve its ability to evaluate genetic testing laboratories. The CAP Laboratory Accreditation Program (LAP) has included cytogenetics laboratories for several years. It is likely that the LAP will include biochemical and molecular genetics in the near future. The CAP Interlaboratory Comparison Program offers a level of proficiency testing through its Surveys. Surveys cosponsored with the ACMG include cytogenetics (CY), biochemical genetics (BGL), and molecular genetics (MGL). None of the tests covered by these surveys is yet listed as a regulated analyte by HCFA regulation. As a result, participation in the Surveys or any other proficiency testing programmes is not required for accreditation under the CAP's LAP. The CAP has recently been granted deeming authority by HCFA. For those laboratories which elect to use the LAP to comply with HCFA regulation, participation in the Surveys is required.

The rapid pace of new developments in clinical genetics also presents new problems for genetics laboratories. Though there is great pressure to make new tests available for diagnostic purposes, implementation is often on an investigational basis because of difficulties in validating the test system or in meeting current regulatory requirements. It is apparent that these difficulties have been recognised by professional organisations and by regulatory agencies. The Laboratory Practice Committee of the ACMG has at least one subcommittee focusing on transferring new tests and technologies from research to clinical practice. Additionally, a subcommittee on quality assurance is drafting guidance for in house validation of DNA probes used for fluorescent in situ hybridisation (FISH) on metaphase chro- 
mosomes. The FDA is reviewing its classification scheme and the requirements for premarket evaluation as they apply to products used for the in vitro diagnosis of genetic disorders. Thus, all current issues of personnel and laboratory practices in genetics originate from a common need for sensitive and reliable diagnostic testing.

This article is the byproduct of deliberation and discourse which culminated in the Laboratory Practice Workshop at the Annual Meeting of the New England Regional Genetics Group (NERGG) on 1-2 December 1994, in Durham, New Hampshire. Dr Mark is the Director of the Laboratory of Cytogenetics, FISH and Genotoxicology at RI Hospital, a memor of enetics, minger Recertification Committee of the Amber of the seven a moderator for the workshop. Dr Kelly is the 1994 President of the American Board of Medical Genetics and an invited speaker at the workshop. Dr Watson is the Chairperson of the Laboratory Practice Committee of the American College of Medical Genetics and an invited speaker at the workshop. Dr Hoeltge is a member of the Cytogenetics Resource Committee of the College of American Pathologists which is responsible for a proficiency testing programme widely subscribed to by cytogenetics laboratories. Dr Hoeltge is also an invited speaker at the workshop. Dr Miller, Director of the Prenatal Diagnostic Center in Lexington, MA and Editor of the Genetic Resource, is an invited participant. Dr Beauregard is the Director of the Genetics Program at the Eastern Maine Medical Center and is an invited participant and co-moderator of the workshop. We wish to thank Ms Freda Yoder of the US Food and Drug Administration for invaluable suggestions and advice, and Ms Margaret A Leoni-Lugo of the I the Laboratory The section of the FDA and relevant portions pertaining to HCFA regulations were based in part on the information provided by Ms Yoder and Ms Leoni-Lugo at the Laboratory Gasparini with Dr Miller's portion of the manuscript. Lastly, Gasparini with Dr Miller's portion of the manuscript. Lastly, Dr Mark wishes to acknowledge the continued support of Dr
Paul H LaMarche, Dr Larry Beauregard, Dr Roger Mark, and the dedicated staff of the Laboratory of Cytogenetics, FISH and Genotoxicology at Rhode Island Hospital on the occasion of the 30th anniversary of their genetic testing laboratory.

1 Moorhead P, Nowell P, Mellman W. Chromosome preparation of leukocytes cultured from human peripheral blood. Exp Cell Res 1960;20:613-6.

2 Guthrie R. Screening for inborn errors of metabolism in the newborn infant: a multiple test program. In: Bergsma D ed. Human genetics. Birth Defects: Original Articles Series Volume IV, No 6. New York: The National FoundationMarch of Dimes, 1968

3 Smith DW. Recognizable patterns of human malformation. Philadelphia: Saunders, 1970, 1976, 1982.

4 American College of Medical Genetics (ACMG). Standards and guidelines: Clinical genetics laboratories. ACMG Laboratory Practice Committee, 1994:1-34.

5 Mark HFL, Watson MS. Evolving standards of practice for clinical cytogenetics. Rhode Island Med 1994;77:373-4.

6 Andrews LB, Fullarton JE, Holtzman NA, Motulsky AG, eds. Assessing genetic risks: implications for health and social policy. Executive Summary of the Institute of Medicine. policy. Executive Summary of the Institute of Medic 7 Yoder F. Federal regulations for diagnostic genetic testing: the $F D A$ perspective. Laboratory Practice Workshop, New Eng-
land Regional Genetics Group Annual Meeting, 1 Deland Regional Genetics Group Annual

8 Hoeltge GA, Dewald G, Palmer C, Miles J. Proficiency testing in cytogenetics; review of the 1986 experience of the College of American Pathologists. Arch Pathol Lab Med 1988;112:1085-90.

9 Hoeltge GA, Dewald G, Palmer CG, et al. Proficiency testing in clinical cytogenetics; a 6-year experience with photographs, fixed cells, and fresh blood. Arch Pathol Lab Med 1993;117:776-9.

10 Harnden DG, Klinger HP, eds. An international system for human cytogenetic nomenclature. Basel: Karger, 1985.

11 Mitelman F, ed. Guidelines for cancer cytogenetics: supplement to an international system for human cytogenetic nomenclature. Basel: Karger, 1991 .

12 American Society for Human Genetics (ASHG). 1994-1995 Guide to North American graduate and postgraduate training pride to North American graduate and postgraduate training

13 Knutsen T, ed. Association of Cytogenetic Technologists (ACT) 1994 international cytogenetic laboratory directory. Burbank, CA: Association of Cytogenetic Technologists, 1994. 